\title{
AGENT-BASED ASSESSMENT OF TRAJECTORY-ORIENTED OPERATIONS WITH LIMITED DELEGATION
}

\author{
Todd J. Callantine, Everett A. Palmer, * Jeff Homola, Joey Mercer, and Thomas Prevot \\ San Jose State University/NASA Ames Research Center, * Moffett Field, CA
}

\begin{abstract}
An evolutionary concept called 'trajectoryoriented operations with limited delegation' is the subject of a preliminary assessment conducted using fast-time simulations with computational agents that represent air traffic controllers. The concept integrates technologies relevant to the Next Generation Air Transportation System (NGATS) and holds promise for efficiency gains by enabling aircraft to fly Continuous Descent Approaches (CDAs). The assessment focuses on how controller strategies and automation tools impact CDA operations. The results indicate the concept represents an advance toward higher-efficiency NGATS operations, and emphasize the importance of shared information and air traffic controller decision support tools.
\end{abstract}

\section{Introduction}

The central theme of the Joint Planning and Develop Office (JPDO) vision for the Next Generation Air Transportation System (NGATS) is the integration of advanced technologies to improve the capacity and efficiency of today's air traffic management (ATM) system without compromising safety [1]. Successful integration of advanced technologies requires careful consideration of the roles and responsibilities of human operators. Operational acceptability, required coordination, strategies for controlling traffic and coping with contingencies, and potential workload of human operators working with advanced technologies must be well understood, even for backup roles.

Research on NGATS concepts and evolutionary pathways for achieving them is currently underway. A concept called 'trajectoryoriented operations with limited delegation' under investigation at NASA Ames Research Center is an evolutionary concept proposed to achieve efficiency and capacity benefits through the combination of time-based traffic flow management, trajectoryoriented operations, and delegation of spacing task to flight crews of appropriately equipped aircraft [2]. Like NGATS concepts planned for implementation two decades from now, this concept integrates new technologies and redefines the roles and responsibilities of human operators in the system.

Researchers have available three general approaches for investigating new air traffic management (ATM) concepts, all of which can yield valuable insights. First, researchers can apply analytical methods to quantify, from a theoretical perspective, expected benefits and risks. Analytical studies are particularly well-suited to examining technological aspects of new concepts. Second, researchers can conduct human-in-the-loop (HITL) studies, in which qualified practitioners work with prototype technologies in realistic settings to gauge the suitability of the technologies and iteratively refine their implementations together with the roles and responsibilities of human operators. HITL studies are invaluable for this purpose, but can be expensive and time-consuming to conduct. The third approach, and the focus of this paper, is to simulate operations across a range of conditions in fast time, using computational agents that represent human operators to perform envisioned roles. This approach holds considerable promise for complementing the other two approaches by identifying, quickly and inexpensively, humansystem interactions that warrant detailed investigation in HITL studies, or providing data to support analytical studies (e.g., safety and risk assessments [3]).

The focus of an agent-based analysis depends in large part on the capabilities of the computational agents. Cognitive human performance models that include models of cognitive processes can provide data on how of human cognitive limitations affect operations [3]. Alternatively, task-analytic worksystem models can identify how environmental effects and new technologies impact nominal task performance. The term 'worksystem' emphasizes 
the use of representations explicitly tied to the ATM domain; 'task-analytic' refers to using explicit computational models of tasks and the operational contexts in which controllers should nominally perform them. While computational agents based on task-analytic worksystem models cannot flexibly respond to every possible ATC context, they can provide a good sense of what is workable.

This paper applies the latter approach to a near-term instantiation of the concept of trajectoryoriented operations with limited delegation. It presents a study to assess air traffic control (ATC) operations across a range of environmental conditions as a complement to upcoming HITL simulations. The paper first describes the concept, and an instantiation of it developed for HITL simulations in the Airspace Operations Laboratory (AOL) at NASA Ames [4]. It then describes the method used in the current research, including traffic scenarios, experimental conditions, a fasttime simulation with simulated automation tools, and computational agents that represent nominal performance of human operators providing ATC services. After presenting the results of the agentbased concept assessment, the paper concludes by discussing implications of the results for HITL studies.

\section{Concept Description}

The idea behind the trajectory oriented operations with limited delegation concept is to achieve overall capacity and efficiency gains at acceptable levels of safety by integrating three facets of operations:

- Time-based flow management to regulate traffic density

- Trajectory-based operations to create efficient, nominally conflict-free trajectories that conform to traffic management constraints

- Airborne separation assistance to maintain local spacing between aircraft.

Since it was first published [2], enabling arrival aircraft to fly efficient Continuous Descent Approaches (CDAs) to their assigned runways has become a primary goal for the concept. It has also been extended to include an important 'arrival flow conditioning' role for participating airline operations centers (AOCs). Participating AOCs use schedule information to issue en route speed clearances to sequence and space arriving company aircraft with the goal of enabling them to fly uninterrupted CDAs. ${ }^{1}$ Controllers are responsible for adjusting the sequencing and spacing of aircraft from non-participating airlines, and for preventing crossing traffic from interfering with aircraft flying CDAs.

Planned HITL explorations will examine the concept in both near-term and farther-term instantiations. In the near-term condition, controllers have only current-day information. In the farther-term conditions, runway schedules are considered to be shared among controllers and participating AOCs. To support the farther-term concept, the following prototype decision support tools have been developed in the AOL [5]:

- Timelines that display runway schedule information

- Trajectory-based trial-planning tools for graphically creating conflict-free trajectories that meet schedule constraints

- Spacing advisories that indicate lead aircraft assignment, and current and advised spacing [6]

- Integrated data link that enables controllers to uplink Flight Management System (FMS)-loadable trajectory clearances and spacing information to aircraft.

The concept has been instantiated for the airspace shown in Figure 1. Four airspace sectors are considered: a terminal area, two high altitude sectors, and a low altitude sector that lies beneath the high altitude sector nearest the terminal area. For HITL simulations, surrounding airspace is managed by confederate 'ghost' controllers.

High-altitude sector controllers descend aircraft arriving from the west on the CDA routes shown in Figure 2. Test traffic scenarios have been developed that include arrival flows in which aircraft from participating airlines constitute the majority of arrivals. Controllers are responsible for fitting other

\footnotetext{
${ }^{1}$ This role could also be performed by an 'area planner' ATC position.
} 


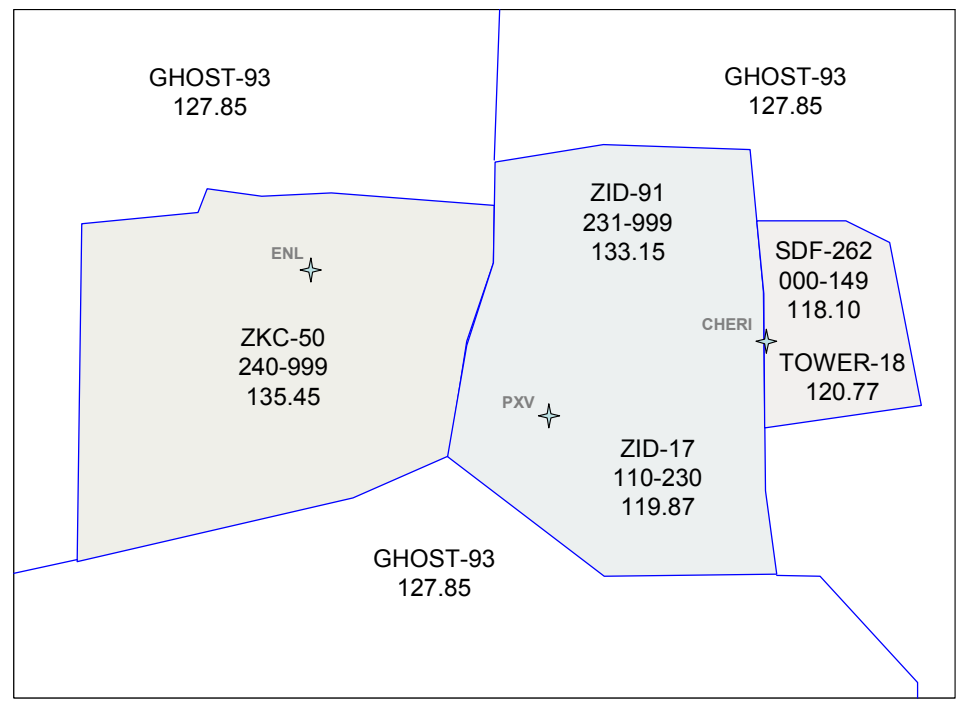

Figure 1. Test Airspace

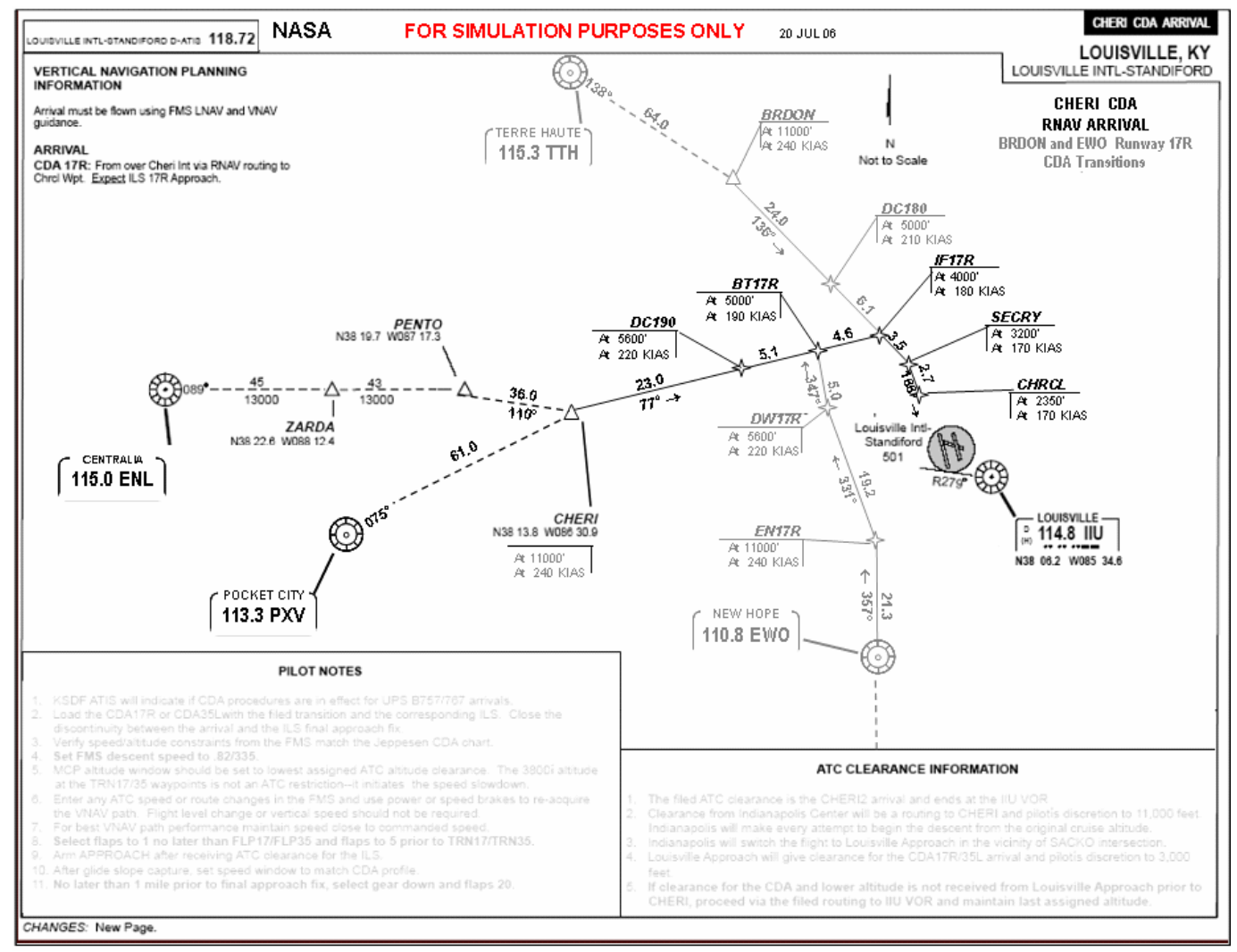

Figure 2. Charted CDA Arrival Transitions for the Test Airspace 
arrivals into the arrival streams. Traffic scenarios also include varying levels of crossing traffic, some of which can interfere with CDA execution if not properly controlled.

Air-ground HITL simulations are currently under development to compare operations with three levels of ground-based automation tool support, in conditions with and without company aircraft from participating AOCs equipped for airborne separation assistance. The first level is current-day operations, in which controllers have no access to schedule timelines or other tools and participating AOCs condition arrival flows. The second level adds shared runway schedules and trajectory-based arrival metering tools; the third level adds the capability for controllers to data link trajectories to appropriately equipped aircraft. The HITL simulations seek to provide capacity data in terms of arrival throughput metrics, efficiency data in terms of potential CDA flight time realized, and safety data related to separation violations, including wake vortex spacing violations at the runway threshold - in addition to data to support iterative concept refinement from a human-systems integration perspective. Important data for this purpose include feedback from pilots and controllers about workload and coordination issues, operational acceptability, coping strategies, and the acceptability and use of prototype automation.

Key issues surrounding air traffic controller roles and responsibilities for the proposed concept include crossing traffic interference with CDA arrivals, and how effectively en route controllers can manage arrivals from non-participating airlines in concert with AOC speed adjustments to company aircraft. This following section describes the elements of a preliminary agent-based assessment of these issues.

\section{Method}

The agent-based assessment focuses on the near-term case in which participating AOCs condition arriving aircraft by issuing speed clearances with the aid of a runway schedule and speed advisory tools while air traffic controllers manage other traffic, and a subset of the fartherterm case in which the runway schedule is shared among AOCs and controllers who also have access to a speed advisory tool. In this assessement, advanced trajectory-based trial planning tools were not considered and all clearances were assumed to be issued by voice. The following subsections describe the fast-time simulation testbed, traffic scenarios, experimental conditions, and agents.

\section{Fast-time Simulation}

The study uses the Trajectory-Centered Simulator (TCSim) [7] to simulate operations in the airspace shown in Figure 1. TCSim enables flexible simulations of trajectory-based operations and includes integrated ATC agents. As yet TCSim does not simulate aircraft equipped for airborne spacing; simulations using agents implemented within the Multi Aircraft Control System (MACS) in the AOL [4] are planned for this purpose.

TCSim also simulates scheduling of arrival aircraft and speed advisory tools. TCSim constructs a schedule by estimating arrival times at a reference fix (here, the runway threshold) and sequencing the aircraft in first-come-first-served order. For runway scheduling, it computes scheduled times of arrival by applying a temporal spacing matrix that specifies inter-arrival times to achieve required wake vortex spacing at the runway threshold. TCSim constructs the schedule such that no aircraft is scheduled before its estimated arrival time. The scheduling process does not account for conflicts arising due to the geometry of merging arrival routes.

TCSim simulates speed advisory automation by predicting arrival times that will result from an aircraft changing its speed to new values within its type-specific speed envelope. Speed values are quantized to the highest precision that is reasonable for speed clearances issued by voice (i.e. 5 knots, $.01 \mathrm{Mach})$. For this research TCSim was configured to produce a speed advisory if it finds a new speed that reduces the offset between the estimated and scheduled arrival times and the new estimated time is not more than five seconds earlier than the scheduled time.

\section{Traffic Scenarios}

TCSim includes facilities for the automatic generation of arrival traffic scenarios, and was used to generate arrival flows with clusters of aircraft arriving on the different routes shown in Figure 2. Some flows were generated to include a small 
scheduling buffer ( 20 seconds); others have densely packed clusters of aircraft. Crossing traffic for the HITL study was created by merging several live traffic recordings from the study airspace. A large number of scenarios were produced by combining TCSim-generated arrival flows with crossing traffic, and twenty were selected for use. Table 1 lists the total number of aircraft in each scenario, the number of arrivals, the number of arrivals from participating AOCs, and the average slack in the arrival schedule (defined as the average of the intervals between scheduled times minus the required temporal spacing values). Shaded scenarios have minimal slack; scenarios listed below the line are considered to have a high level of arrivals from participating AOCs.

Table 1. Traffic Scenarios

\begin{tabular}{|l|l|l|l|l|}
\hline $\begin{array}{l}\text { Scen. } \\
\text { Num. }\end{array}$ & $\# \mathbf{A} / \mathbf{C}$ & $\begin{array}{c}\# \\
\text { Arrs. }\end{array}$ & $\begin{array}{c}\# \\
\text { AOC } \\
\text { Arrs. }\end{array}$ & $\begin{array}{c}\text { Avg. } \\
\text { Slack } \\
\text { (secs) }\end{array}$ \\
\hline 11 & 161 & 26 & 3 & 46.85 \\
\hline 17 & 171 & 36 & 3 & 13.08 \\
\hline 7 & 161 & 26 & 4 & 55.25 \\
\hline 8 & 133 & 26 & 5 & 55.93 \\
\hline 20 & 171 & 36 & 5 & 33.62 \\
\hline 12 & 133 & 26 & 6 & 53.15 \\
\hline 18 & 143 & 36 & 7 & 13.36 \\
\hline 4 & 161 & 26 & 7 & 62.15 \\
\hline 3 & 133 & 26 & 8 & 49.6 \\
\hline 19 & 143 & 36 & 9 & 28.92 \\
\hline 16 & 143 & 36 & 10 & 12.08 \\
\hline 10 & 133 & 26 & 22 & 53.42 \\
\hline 2 & 133 & 26 & 22 & 59.59 \\
\hline 6 & 161 & 26 & 22 & 58.46 \\
\hline 1 & 161 & 26 & 22 & 59.18 \\
\hline 9 & 161 & 26 & 23 & 50.04 \\
\hline 5 & 133 & 26 & 24 & 49.89 \\
\hline 13 & 143 & 36 & 31 & 12.3 \\
\hline 14 & 171 & 36 & 31 & 9.11 \\
\hline 15 & 171 & 36 & 32 & 5.57 \\
\hline & & & & \\
\hline
\end{tabular}

Shorter scenarios were approximately ninety minutes in length; longer ones lasted approximately 110 minutes. Each took between three and four minutes to simulate in TCSim, depending on the processing overhead associated with agent and advisory tool computations. Figure 3 shows the average traffic counts produced in each of the study airspace sectors shown in Figure 1 over time. In certain scenarios the maximum number of aircraft in the ZKC_50 and ZID_91 sectors reaches 24higher than the traffic levels typical of current operations. Aircraft arriving at the end of scenarios with a large number of arrivals contribute to the peak in SDF_262 traffic.

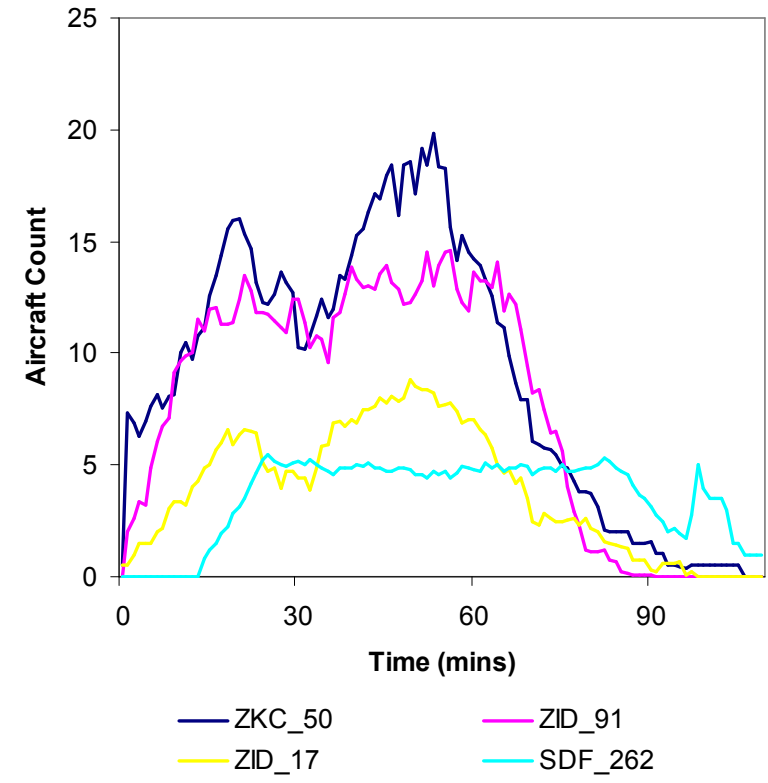

Figure 3. Average Sector Aircraft Counts

The CDA routes comprising the traffic flows also vary across scenarios. Arrivals may be assigned to the ENL-CHERI CDA, PXV-CHERI CDA, or the BRDON or EWO transitions to the CHERI CDA, as shown in Figure 2. Each scenario has at least four BRDON and four EWO arrivals merging with the primary flow from CHERI. The CHERI arrivals either come mostly from ENL, mostly from PXV, or are evenly distributed. This variation affects the number of merges that occur at CHERI in the ZID_17 low-altitude sector. Smallangle merges also occur at ENL and PXV in the ZKC_50 high-altitude sector.

\section{Conditions}

The assessment examined five test conditions (Table 2). The first condition (AOC) is used as a baseline traffic condition in which participating AOCs condition company arrivals by issuing advised speeds. The AOC_AGTS condition adds ATC agents that work all the other traffic using 
current-day control methods. The AOC AGTS TOOLS condition adds a runway schedule that is shared with the AOCs and the capability to issue speed advisories to non-AOC arrivals. The last two conditions (AOC_AGTS_WINDS and AOC_ AGTS_TOOLS_WINDS) are the same as the previous two, except that TCSim creates the schedule and generates requested speed advisories using forecast winds that are ten knots stronger and from a direction ten degrees different from the actual winds at each specified altitude.

Table 2. Test Conditions

\begin{tabular}{|l|l|}
\hline Condition & \multicolumn{1}{|c|}{ Description } \\
\hline AOC & $\begin{array}{l}\text { Participating AOCs } \\
\text { condition company aircraft }\end{array}$ \\
\hline AOC_AGTS & $\begin{array}{l}\text { AOC role, plus ATC } \\
\text { agents controlling traffic } \\
\text { using 'current-day' } \\
\text { methods }\end{array}$ \\
\hline $\begin{array}{l}\text { AOC_AGTS } \\
\text { TOOLS }\end{array}$ & $\begin{array}{l}\text { AOC role, plus ATC } \\
\text { agents using shared } \\
\text { runway schedules and } \\
\text { speed advisory tools }\end{array}$ \\
\hline $\begin{array}{l}\text { AOC_AGTS } \\
\text {-WINDS }\end{array}$ & $\begin{array}{l}\text { AOC_AGTS, with } \\
\text { predicted winds different } \\
\text { from actual winds }\end{array}$ \\
\hline $\begin{array}{l}\text { AOC_AGTS } \\
\text { TOOLS_WINDS }\end{array}$ & $\begin{array}{l}\text { AOC_AGTS_TOOLS, } \\
\text { with different predicted } \\
\text { winds }\end{array}$ \\
\hline
\end{tabular}

\section{ATC Agents}

A computational air traffic controller agent representing a single radar ('R-side') controller is implemented for each of the test sectors. The air traffic controller agents are implemented in the Java programming language within TCSim. This enables each ATC agent to access information on the traffic display and issue clearances to aircraft. In TOOLS conditions, agents query the automation tools simulated in TCSim for information about sequence, schedule, and advised speeds.

The agent model is actually a collection of models that enable it to perform ATC functions. Individual models include:

- High-level executive model to control processing (includes timing information)
- Model of controller's 'picture' and the processes required to construct and maintain it

- Sector-specific knowledge model (including the agent's 'area of regard' and sector exit conditions)

- Model of control strategies to apply

- Models for implementing individual control methods

- Agenda formulation/task management model

- Models of coordination with other agents (including handoffs and communication of clearance information)

- Activity timing model (nominal time to complete each activity)

- Models of decision support tool usage (including times to execute activities using tools).

Figure 4 shows hierarchical process flow diagram for an ATC agent. At left are the three activities represented in the high-level executive model. These activities are performed cyclically. As has been hypothesized for human air traffic controllers, updating the picture constitutes the bulk of processing.

The picture contains everything an agent knows about the current traffic situation, including all its predictions about where individual aircraft are going, as well as predictions about where they should go if the agent should issue certain clearances. These predictions are referred to as planned trajectories ('Planned Trajs') in Figure 4. Planned trajectories enable the agent to determine whether an aircraft is in conflict with other aircraft and the urgencies associated with initiating and accepting handoffs and issuing descent, approach, and landing clearances. An ATC agent generates planned trajectories using heuristics of the sort a human controller might use to predict an aircraft's future trajectory. Most notably, an aircraft's current groundspeed is used for speed estimation. This means that planned trajectories for aircraft in descent have inherent inaccuracies. 


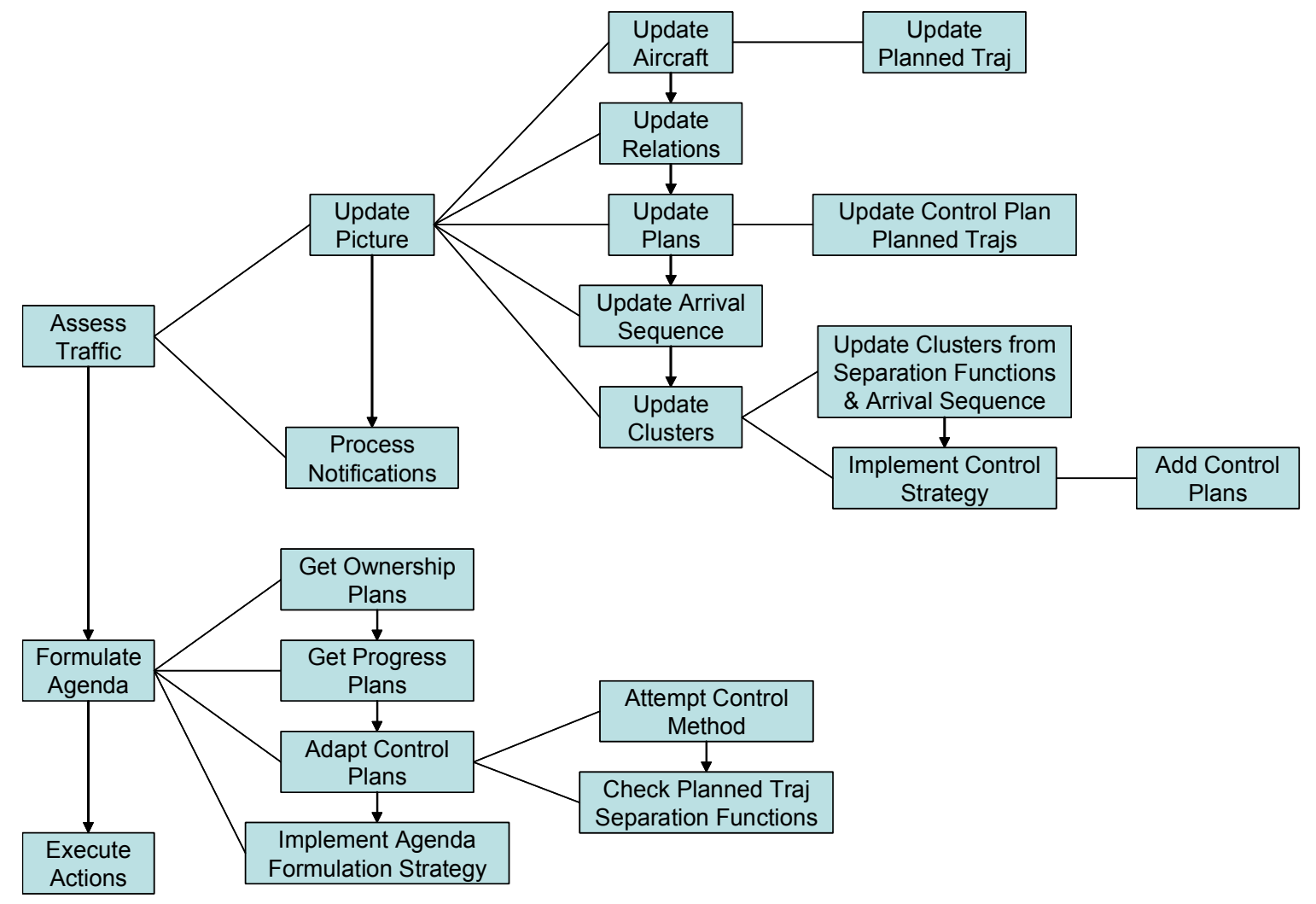

Figure 4. ATC Agent Hierarchical Process Flow Diagram

Aircraft in conflict form a 'cluster' which is used to represent information about the conflict. Implementing a control strategy to address the conflict results causes a control plan to be added to the cluster. The control plan represents a plan to issue a clearance an aircraft, specifying which aircraft to try to clear and the type of clearance to try to issue. An agent does not immediately issue a clearance; instead, the agent waits until it performs the 'formulate agenda' process to collect all the plans that represent activities that need to be performed for each aircraft. At this time it attempts to formulate a specific clearance that implements the control method specified in the control plan.

Table 3 lists an example control strategy. A particular control strategy specifies priorities for choosing which aircraft in a cluster to clear, along with the priorities for trying particular types of clearance. To implement a lateral control method, an agent first tries to issue a clearance direct to down-path waypoints by generating and testing planned trajectories until one is found that will not conflict with the planned trajectories of other aircraft for the duration of the planned trajectory (three minutes into the next sector or ten minutes, whichever is less). Failing this, an agent performs the same process for possible heading vectors.

Altitude control methods are either cruise altitude changes or, if the aircraft is climbing or descending, temporary altitude clearances. If it generates a planned trajectory that solves the conflict, the agent adds the action to issue the clearance to its current agenda.

Table 3. Example Control Strategy

\begin{tabular}{|c|c|c|}
\hline Conflict & Aircraft & Control Methods \\
\hline $\begin{array}{c}\text { Arrival- } \\
\text { Arrival }\end{array}$ & Trail & Speed, Lateral, Vertical \\
\hline $\begin{array}{c}\text { Arrival- } \\
\text { Other }\end{array}$ & Other & Vertical, Lateral, Speed \\
\hline $\begin{array}{c}\text { Other- } \\
\text { Other }\end{array}$ & Either & Vertical, Lateral, Speed \\
\hline
\end{tabular}


In the current implementation, an agent repeatedly tries to apply the highest priority control method specified by its control strategy, until the predicted time to the conflict becomes three minutes or less. The agent then tries other methods specified in the control strategy in turn in an effort to find a workable clearance.

The last step in an agent's 'formulate agenda' process is to apply its agenda formulation strategies to prioritize all the activities that appear on its 'short list' for this cycle. An agenda formulation strategy that places a high priority on transferring control of aircraft, for example, could cause clearances for other aircraft to be left off the final agenda for the current cycle. Planned trajectories for clearances left off the final agenda must be regenerated and checked against other planned trajectories again on a later cycle before the agent can issue the clearance.

In the concept assessment, the ZKC_50 and ZID_91 agents used the control strategy shown in Table 3 in all conditions. The ZID_17 agent, however, used it only in the TOOLS conditions in order to exercise the capability to issue advised speeds to manage arrival-arrival conflicts. In conditions without tools, the ZID 17 agent employed a different strategy that specified lateral clearances as the highest priority for addressing arrival-arrival conflicts. This enables the ZID 17 agent to solve conflicts at the CHERI merge point using heading vectors as observed in current-day operations.

While the SDF 262 agent was operational and assumed control of aircraft, it did not work traffic as it might in a fully functional implementation. Some key capabilities - such as the capability to reassign aircraft to a parallel runway if merges are not working out-are as yet unimplemented. However, having the SDF_262 agent not work terminal-area merge problems affords an opportunity to examine how successfully upstream agents and participating AOCs can condition the arrival flow.

\section{AOC Functionality}

The role of participating AOCs in conditioning arrival flows by assigning speed advisories to aircraft in cruise is automated in TCSim. Once per minute of simulation time, the AOC automation checks aircraft within three hundred nautical miles of the runway for conformance with their scheduled arrival time. If possible it issues a new advised speed to those whose estimated and scheduled times of arrival differ by more than fifteen seconds. The three hundred mile range means AOC aircraft have typically performed any required speed changes before they enter the test airspace. For aircraft scheduled at their wake vortex separation with no extra buffer, the fifteen second tolerance means a slight speed adjustment may still be required at some point before landing.

\section{Flight Crew Functions}

In this study, flight crews are modeled to perform assigned maneuvers after a nominal delay. More advanced flight crew functionality, such as variable compliance timing or random nonconformance, is reserved for later investigations.

\section{Data Collection}

Each scenario was simulated once under each condition for a total of one hundred trials. TCSim produced data on inter-arrival spacing, clearances the agents issued, and any separation violations that occurred. The next section describes the results of the agent-based concept assessment.

\section{Results}

Data analysis focused on crossing traffic interference with CDA arrivals, and how effectively en route controllers can manage arrivals from nonparticipating airlines when participating AOCs have applied speed adjustments to company aircraft. As noted above, the analysis focuses on providing a sense of what is generally workable, recognizing that the ATC agents cannot flexibly respond to every possible context.

As a gauge of how well the ATC agents control traffic, Figure 5 shows the agents' effectiveness in maintaining separation between individual over-flights and departures (i.e., nonarrival aircraft). All conditions with agents show a significant reduction in the average number of observed violations from the AOC condition. (Error bars represent one standard deviation.) Neither the amount of slack in the schedule nor the level of AOC involvement appears to affect these results. A 


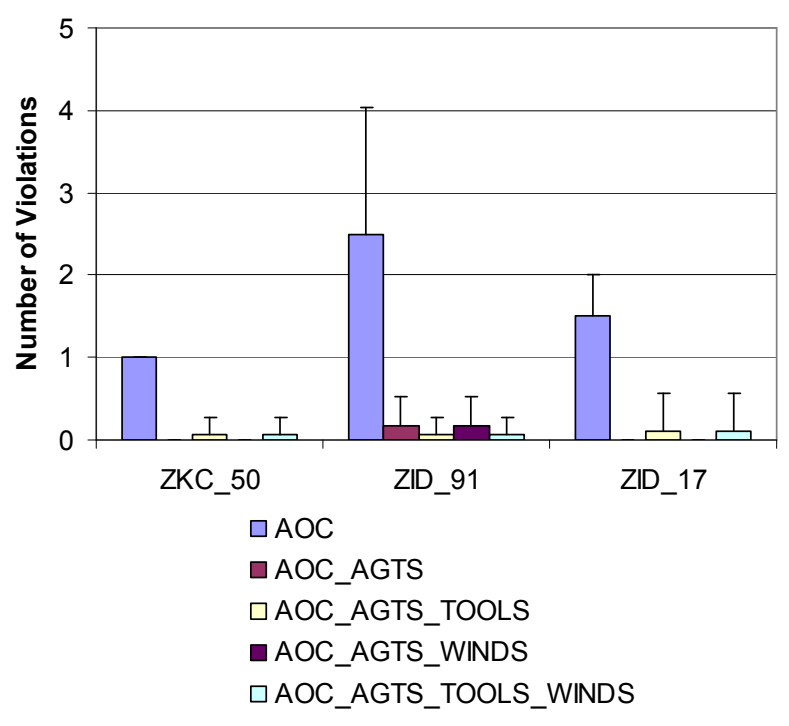

Figure 5. Average Number of Separation Violations between Non-Arrival Aircraft per Scenario by En Route Sector for All Scenarios

preliminary analysis indicates that some violations were essentially 'handed to' the en route agents from the surrounding ghost sectors, because traffic in the ghost sectors is not controlled and the ATC agents accept all handoffs.

Against this backdrop, Figure 6 reflects the effectiveness of the agents in preventing interference between arrivals and crossing traffic. It shows the total number of loss of separation events that occurred between an arrival aircraft and a departure or over-flight in the ZKC_50, ZID_91, and ZID_17 sectors under all conditions. Agents successfully prevented conflicts between arrivals and other aircraft by moving crossing traffic out of the way of the arrival flows, as specified by their control strategies (Table 3). Although the control strategies specified that altitude clearances were preferred for this purpose, lateral clearances were issued in significantly greater numbers. This suggests that flight levels in the test scenarios were densely populated.

The data suggest that, in conditions without tools, agents perform somewhat better in scenarios with a high level of AOC involvement (i.e. scenarios above the line in Table 1). For example, nine out of the ten violations for the ZID_91 agent in the AOC_AGTS and AOC_AGTS_WINDS

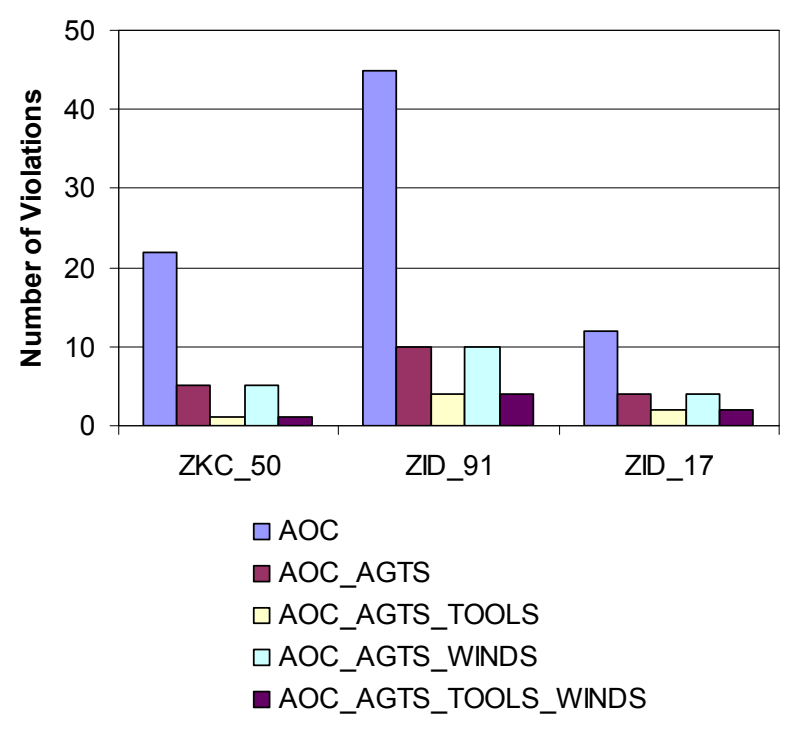

\section{Figure 6. Total Number of Separation Violations for All Scenarios between Arrivals and Other Aircraft by En Route Sector}

conditions shown in Figure 6 occurred in scenarios with low numbers of AOC-conditioned arrivals. Problems in other sectors were evenly divided between high and low levels of AOC involvement. Any trend toward worse performance in scenarios with low numbers of AOC aircraft is most likely an artifact of agent performance degradation when managing large numbers of aircraft.

As the arrival flows compress, more opportunities for violations between arrivals occur. The highest potential is in the SDF_262 sector. However, this is also where the terminal-area merges are most likely to create problems. Because the SDF_262 agent lacked key capabilities for managing merges, it is somewhat misleading to examine all arrival-arrival interactions. Figure 7 therefore presents totals for terminal-area arrivalarrival interactions that involved only CHERI arrivals. No obvious effects of AOC involvement or schedule slack are apparent in the data. (Because effects due to the differences between forecast and actual winds were negligible for all the data examined, these and subsequent results focus on the AOC, AOC_AGTS, and AOC_AGTS_TOOLS conditions.)

The results shown in Figure 7 are interesting because the total number of violations in the $\mathrm{AOC}_{-}$ 


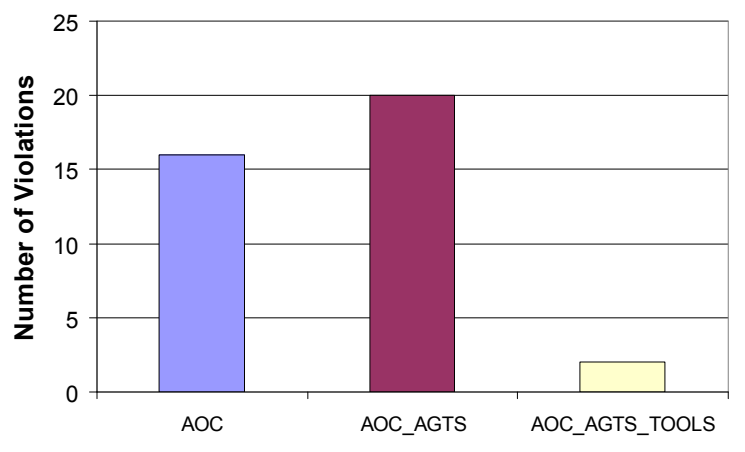

Figure 7. Total Number of Terminal-Area Separation Violations (CHERI Arrivals Only)

AGTS condition exceeds the number in the AOC condition. This indicates that shared schedule information, and the capability to control to scheduled arrival times using speed advisories, is important for enabling uninterrupted CDA arrivals. Without these tools, upstream agents are left to use speeds (per their control strategies) for relative spacing adjustments, which may not jibe with AOC adjustments - let alone aircraft that, unbeknownst to them, will later be merging with the flow.

More evidence about the importance of shared schedule information is provided by data on spacing accuracy of the arrivals at the runway threshold. Figure 8 depicts spacing accuracy histograms, plotted as lines, for the case when low numbers of arrivals are conditioned by participating AOCs. Again recognizing that the results largely reflect how well the flows are conditioned for merging and spacing by the upstream agents, Figure 8 shows the schedule information and speed advisory tools are essential. With tools the agents produce relatively well-spaced arrivals; without them the agents fail to improve the accuracy achieved in the AOC condition. Even in the TOOLS conditions, aircraft not conditioned by AOC arriving from BRDON and EWO can cause problems because speed control authority is diminished in the terminal area. (The histogram in Figure 8 is limited to between -40 and 40 seconds of spacing error. Schedule slack skews the results toward excess spacing.)

Figure 9 depicts spacing accuracy for the case when a large number of arrivals are conditioned by participating AOCs. In the absence of tools, the agents essentially undo what the AOCs have done, because they lack a shared understanding of the sequence and schedule. With tools, however, the agents are able to fit non-AOC arrivals into the flow, yielding a slight improvement in spacing accuracy over the AOC condition. (The histogram in Figure 9 is also limited, giving an impression of symmetry when in fact it is also skewed toward excess spacing.)

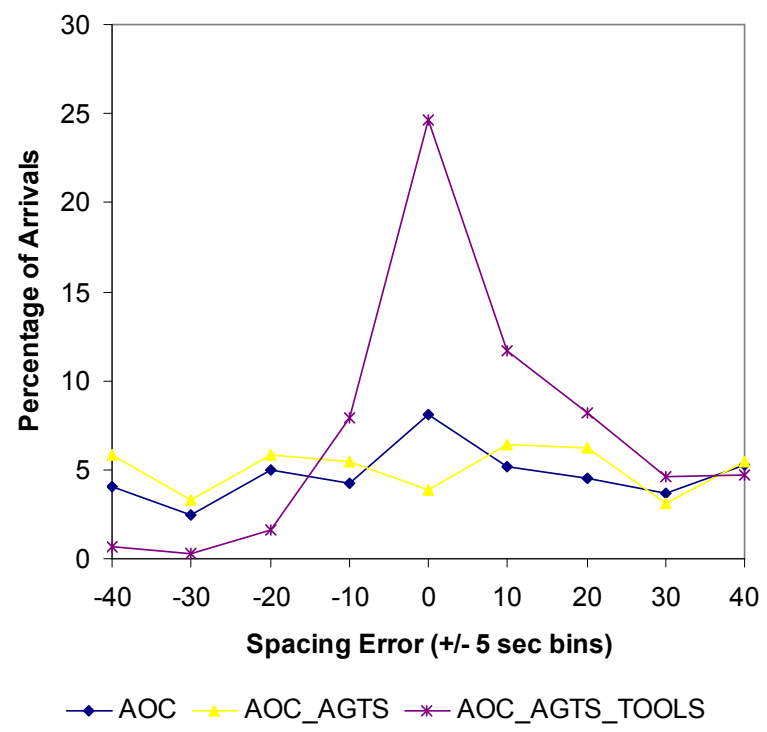

Figure 8. Spacing Accuracy Histogram for Scenarios with Low AOC Involvement

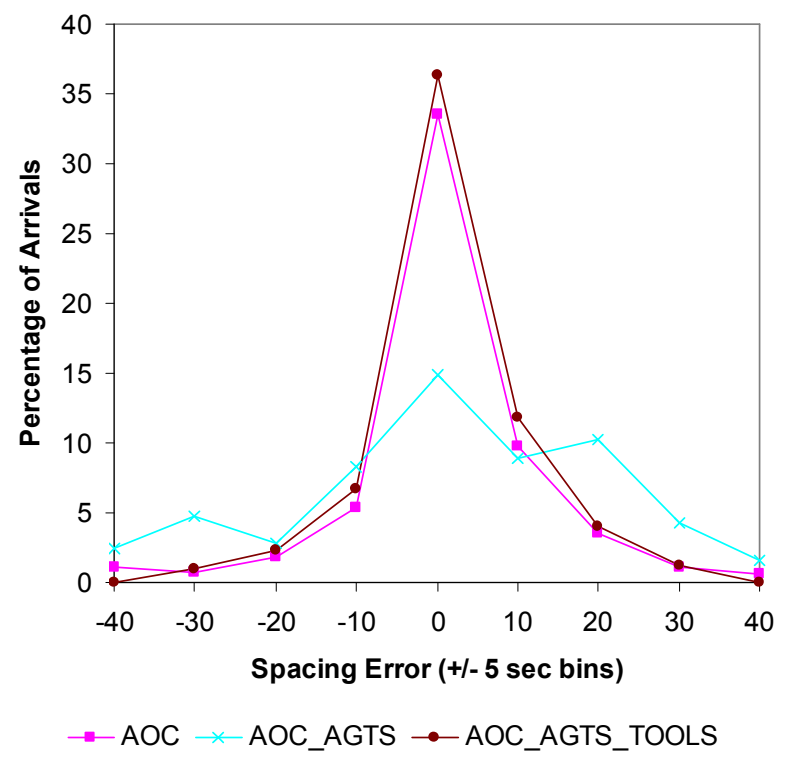

Figure 9. Spacing Accuracy Histogram for Scenarios with High AOC Involvement 
An examination of clearances issued to arrival aircraft showed that, true to its control strategy, the ZID_17 agent issued some heading vectors to arrival aircraft in the AOC_AGTS condition that would likely result in the interruption of a CDA. In the AOC_AGTS_TOOLS condition, however, the ZID_17 agent issued only a few speed clearances. SDF_262 also only issued a few speeds. Overall, the number of heading and altitude clearances with the potential to interrupt CDAs was greatly reduced in the conditions with tools.

\section{Conclusion}

The results of the assessment reflect favorably on the trajectory-oriented operations with limited delegation concept when tools are available and scheduling information is shared with participating AOCs - even in relatively high traffic conditions with dense arrival flows. Human air traffic controllers using suitable shared scheduling and advisory tools with participating AOCs should be capable of enabling uninterrupted CDAs for the vast majority of arrivals, particularly if the runway schedule includes any degree of slack. In fartherterm instantiations of the concept, trajectory-based trial-planning tools and data link technologies should enable controllers to perform lateral route adjustments to make small adjustments to the arrival flow without interrupting CDAs, and aircraft equipped for airborne spacing stand to further reduce the need for disruptive clearances.

HITL studies will help understand the balance between workload associated with monitoring AOC adjustments to arrivals and workload associated with conditioning other arrivals and ensuring separation. These results suggest human air traffic controllers are likely to find AOC participation acceptable as long as AOCs consistently condition aircraft for arrival early and well. How AOC speed commands affect controllers in the sectors in which they occur is a topic that also deserves examination. HITL studies will afford the opportunity to refine the concept, and transfer lessons learned about preferable control strategies to agent models.

\section{Acknowledgments}

Funding support for this research is provided by the NASA Airspace Systems Program.

\section{References}

[1] JPDO, 2006, Concept of Operations for the Next Generation Air Transportation System, Draft version 0.2, July 24, 2006.

[2] Prevôt, T., T. Callantine, P. Kopardekar, N. Smith, E. Palmer, V. Battiste, 2004, TrajectoryOriented Operations with Limited Delegation: An Evolutionary Path to NAS Modernization, AIAA2004-6449, American Institute of Aeronautics and Astronautics, Reston, VA.

[3] Blom H., K. Corker, and S. Stroeve, 2005, On the Integration of Human Performance and Collision Risk Simulation Models of Runway Operation, Proceedings of the 6th USA/Europe Air Traffic Management Research and Development Seminar, Baltimore, MD..

[4] Prevot, T., N. Smith, E. Palmer, J. Mercer, P. Lee, J. Homola, T. Callantine, 2006, The Airspace Operations Laboratory (AOL) at NASA Ames Research Center, AIAA-2006-6112, American Institute of Aeronautics and Astronautics, Reston, VA.

[5] Prevot, T., V. Battiste, T. Callantine, P. Kopardekar, P. Lee, J. Mercer, E. Palmer, N. Smith, 2005, Integrated Air/Ground System: TrajectoryOriented Air Traffic Operations, Data Link

Communication, and Airborne Separation Assistance, Air Traffic Control Quarterly, Vol. 13, No. 2, pp. 201-229.

[6] Callantine, T., P. Lee, J. Mercer, T. Prevôt, E. Palmer, 2005, Simulation of Terminal-Area Flight Management System Arrivals with Airborne Spacing, Air Traffic Control Quarterly, Vol. 14, No. 1, pp. 47-67.

[7] Callantine, T., 2004, Modeling and Simulation of Trajectory-Oriented Air Traffic Management Concepts, AIAA-2004-5260, American Institute of Aeronautics and Astronautics, Reston, VA.

\section{Email Address}

tcallantine@mail.arc.nasa.gov

\section{$25^{\text {th }}$ Digital Avionics Systems Conference}

October 15, 2006 